\title{
A segurança alimentar e nutricional e o uso da abordagem de direitos humanos no desenho das políticas públicas para combater a fome e a pobreza ${ }^{1}$
}

\section{(}

ENSAIO | ESSAY

\section{Food and nutritional security and the use of a human}

rights-based approach on the development of

public policies to fight hunger and poverty

Maria de Fátima Machado de ALBUQUERQUE²

R E S U M O

Este ensaio tem como objetivo enfatizar a importância da utilização da abordagem de direitos humanos no desenho das políticas públicas para combater a fome e a pobreza no Brasil. Inicialmente foi apresentado um marco teórico sobre o conceito de segurança alimentar e nutricional e o direito humano à alimentação adequada. Também foram identificadas as obrigações do Estado para a realização do direito humano à alimentação adequada, bem como o que se identifica como potenciais violações deste direito. Depois se buscou discutir o significado de uma abordagem baseada nos direitos e como esta abordagem pode contribuir para a promoção do direito à alimentação adequada. Foi realizada uma breve reflexão sobre a questão da (in)segurança alimentar, suas diversas formas de avaliação e sobre a importância de investigar mais detalhadamente as repercussões desta questão sobre o indivíduo e a família, inclusive daquelas que participam de programas sociais.

Termos de indexação: Direitos humanos. Políticas públicas. Segurança alimentar e nutricional.

\section{A B S T R A C T}

This essay aims to discuss the meaning of a human rights-based approach in the context of the public policies used in the fight against hunger and poverty in Brazil. Some aspects were presented such as the accepted concepts of food and nutritional security and the human right to appropriate nutrition. The obligations of the State to fulfill the human right to appropriate nutrition were also identified as well as that

\footnotetext{
${ }_{1}$ Artigo elaborado como parte do projeto de pós-doutorado da autora no Norwegian Centre for Human Rights, University of Oslo.

2 Universidade Federal de Alagoas, Faculdade de Medicina. Av. Lourival Melo Mota, s/n., Campus A.C. Simões, Tabuleiro do Martins, 57072-900, Maceió, AL, Brasil.E-mail:<fatimamachado@uol.com.br>.
} 
896 | M.F.M. ALBUQUERQUE

which is seen as potential violations of this right. Then, the meaning of a rights-based approach and how this approach can contribute to promote the right to appropriate nutrition were discussed. A concise reflection was done on the food (in)security issue, its many ways of assessment and the importance of investigating in a more detailed manner the repercussions of this issue on individuals and families, including those who are benefited by social programs.

Indexing terms: Human rights. Public policies. Food security.

\section{N T R O D U ÇÃ O}

A construção do conceito sobre Segurança Alimentar e Nutricional (SAN) tem revelado, a partir dos intensos debates ocorridos especialmente nas últimas décadas, as diversas dimensões que compõem este conceito e como estas se inter-relacionam com a realização do Direito Humano à Alimentação Adequada (DHAA) e com a luta contra a fome, a pobreza e as diferentes formas de desigualdade, especialmente nos países em desenvolvimento, a exemplo do Brasil.

De uma forma mais geral, a SAN pode ser entendida como sendo a base e também um dos parâmetros contextuais para a realização do DHAA, apesar de a efetivação deste direito ser independente da existência da SAN ${ }^{1}$.

\section{Segurança alimentar e nutricional e o direito à alimentação adequada}

Historicamente, a inter-relação entre a SAN e o DHAA começou a se desenhar a partir do entendimento do que seria a constituição dos direitos humanos na Declaração Universal dos Direitos Humanos, em 19482,3. Naquela época, a principal preocupação foi enfatizar a noção de que os seres humanos, enquanto indivíduos pertencentes a uma sociedade, tinham direitos e que estes direitos deveriam ser reconhecidos e expressos nas diversas dimensões das quais faziam parte.

A grande contribuição da declaração foi inserir a proposta de que, para a efetivação dos direitos, seria necessária a inclusão das questões sociais, econômicas, civis e políticas, as quais foram essenciais para a identificação destas, enquanto direitos vinculados às liberdades fundamentais $\mathrm{e}$ à dignidade humana.
À luz desta perspectiva, o direito humano à alimentação adequada foi reconhecido em 1966, a partir do Pacto Internacional para os Direitos Econômicos, Sociais e Culturais (PIDESC), quando 146 países reafirmaram e ratificaram seu reconhecimento ${ }^{2-4}$.

Entretanto, foi em 1996 durante a Cúpula Mundial de Alimentação (CMA) em Roma, que chefes de estado e governos, empenharam a sua vontade política e asseveraram "o direito fundamental de estar livre da fome". Tal indicação culminou em um Plano de Ação que apontava sete compromissos, dentre os quais, os de assegurar um ambiente político, social e econômico para viabilizar melhores condições para a erradicação da pobreza e a implementação de políticas voltadas à erradicação da pobreza e da desigualdade e para a promoção da segurança alimentar e sustentável para todos ${ }^{2}$.

Um dos encaminhamentos da Cúpula Mundial de Alimentação foi a solicitação, ao Comitê de especialistas das Nações Unidas para os Direitos Econômicos, Sociais e Culturais, da definição do conceito e das formas de operacionalização do Direito Humano à Alimentação Adequada. Em razão dessa demanda, o Comitê elaborou o Comentário Geral (CG) $n^{\circ} 12^{5}$, que traz uma interpretação acerca do artigo 11, parágrafos 1 e 2, do PIDESC:

O direito à alimentação adequada realiza-se quando cada homem, mulher e criança, sozinho ou em companhia de outros, tem acesso físico e econômico, ininterruptamente, à alimentação adequada ou aos meios para sua obtenção. O direito à alimentação adequada não deverá, portanto, ser interpretado em um sentido estrito ou restritivo, que o equaciona em termos de um pacote mínimo de calorias, 
proteínas e outros nutrientes específicos. O direito à alimentação adequada terá de ser resolvido de maneira progressiva. No entanto, os estados têm a obrigação precípua de implementar as ações necessárias para mitigar e aliviar a fome, como estipulado no parágrafo 2 do artigo 11, mesmo em épocas de desastres, naturais ou não.

Duas contribuições ficaram mais definidas ao longo desse processo: o reconhecimento do provimento dos direitos como obrigação do Estado e a inter-relação do direito humano à alimentação adequada com o conceito mais amplo de segurança alimentar, uma vez que sua realização não é somente relacionada ao provimento do alimento, mas a uma abrangência mais ampla que contempla outros direitos, especialmente quando se considera a assertiva de que os direitos humanos são universais, indivisíveis, inalienáveis, interdependentes e inter-relacionados ${ }^{2,3}$.

O conceito de SAN utilizado no Brasil, o qual é oriundo das conferências nacionais de segurança alimentar e fruto de grande mobilização de organizações da sociedade civil, está definido na Lei Orgânica de Segurança Alimentar e Nutricional (LOSAN) ${ }^{6}$, sancionada em setembro de 2006, como "a realização do direito de todos ao acesso regular e permanente a alimentos de qualidade, em quantidade suficiente, sem comprometer o acesso a outras necessidades essenciais, tendo como base práticas alimentares promotoras da saúde, que respeitem a diversidade cultural e que sejam social, econômica e ambientalmente sustentáveis". Além disso, a LOSAN também reconhece e estabelece que "a alimentação adequada é um direito fundamental do ser humano, inerente à dignidade da pessoa humana e indispensável à realização dos direitos consagrados na Constituição Federal, devendo o Poder Público adotar as políticas e ações que se façam necessárias para promover e garantir a Segurança Alimentar e Nutricional da população". E é nesse sentido que Freitas \& Pena ${ }^{7}$ destacam que "a SAN deve ser vista como um conjunto de direitos e políticas sociais contidas na noção de seguridade social inscrita no Artigo 194 da Constituição do Brasil".

As implicações destes conceitos para a realidade do País é que estabelecem, não somente a inter-relação da Segurança Alimentar e Nutricional com a realização do Direito Humano à Alimentação Adequada e com a luta contra a fome, a má-nutrição e pela garantia da alimentação adequada para todos, mas também definem claramente as obrigações do governo brasileiro frente a estas demandas. Outro aspecto relevante foi que a normatização do DHAA pela LOSAN abriu espaço para que fosse possível a exigibilidade do DHAA no Brasile-10.

\section{Obrigações do governo em relação à realização dos direitos humanos, em especial o DHAA}

A partir da Declaração de 1948 iniciou-se um processo de universalização dos direitos humanos, que propiciou a formação de um sistema internacional de proteção desses direitos, mediante a adoção de vários tratados internacionais ${ }^{11}$.

Quando um país ratifica uma convenção ou tratado internacional, que tem a característica de ser legalmente vinculante, como é o caso do PIDESC, ele se compromete internacionalmente, enquanto Estado-parte, em assumir sua obrigação de realizar os direitos e de não os violar ${ }^{12,13}$. A natureza geral das obrigações possui algumas categorias $^{12}$, sendo a primeira respeitar, a segunda proteger e a terceira realizar (promover e prover) os direitos, de forma diligente e eficaz. A obrigação de respeitar demanda que o Estado não tome quaisquer medidas que interfiram ou bloqueiem, direta ou indiretamente, a realização dos direitos. Entretanto, se o fizer, terá que criar mecanismos de reparação. A segunda obrigação requer que o Estado proteja os habitantes de seu território contra ações de terceiros (empresas ou indivíduos), que possam resultar em violações aos direitos, e 
também adotar medidas preventivas para que tais ações não aconteçam. Quanto à obrigação de realizar, o Estado deve promover/facilitar o acesso de pessoas a recursos e meios, de forma a garantir a plena fruição de seus direitos.

Esta mesma estrutura se aplica às obrigações relativas ao DHAA. Para tanto, o Estado deve respeitar, proteger o DHAA e também promover/facilitar políticas e programas públicos, recursos e meios que garantam o DHAA para todos. Além disso, está contido nessa obrigação, o ato de prover alimentação e nutrição, com dignidade, de famílias que passam fome ou estão desnutridas, por condições que fogem ao seu controle 5 .

Um aspecto adicional a ser considerado em relação à especificidade do DHAA, é a questão da realização progressiva. A fome é uma condição que necessita uma intervenção imediata porque ultraja a dignidade humana, por isso sua erradicação deve ser considerada como prioridade na agenda política de qualquer governo. E, se por um lado, o termo realização progressiva pode ser interpretado como um direito que não será alcançado em um breve espaço de tempo, por outro, deve ser entendido também que o Estado tem a obrigação de avançar o mais diligente e efetivamente possível para alcançar aquela meta. Além disso, e à luz desta compreensão, qualquer medida originada pelo desempenho de uma política pública, que promova um retrocesso ou um impacto negativo na promoção de um direito, pode ser considerada como uma falha na obrigação do poder público em prover determinado direito ${ }^{14}$.

\section{Violações dos direitos e a perspectiva do DHAA}

Compreende-se por violação dos direitos humanos, toda a situação/condição em que não haja cumprimento dos princípios de respeito à dignidade humana, ou seja, a não efetivação dos direitos civis, políticos, econômicos, sociais e culturais $^{11,15}$. Considerando a complexa realidade brasileira, marcada por um modelo de desenvolvimento gerador de profundas desigualdades, exclusão social e pobreza, pode-se dizer que as violações de direitos existentes (inclusive no que diz respeito ao DHAA) são um construído histórico, que necessita urgência na adoção de medidas e ações eficazes para a correção das violações e para a promoção dos direitos ${ }^{11}$.

Na ótica dos direitos humanos, o conceito de violação também está relacionado à falha do poder público, por meio de uma ação direta, em cumprir suas obrigações legais de respeitar, proteger, promover e prover os direitos. Além disso, existem também várias omissões realizadas pelo poder público que contribuem para a violação do DHAA $^{9}$.

O Brasil ratificou o PIDESC em 1992 e consubstanciou um avanço normativo relativo ao DHAA com a elaboração da LOSAN, em 2006. Por isso, mais do que nunca, o Estado brasileiro tem de avançar na realização prática do DHAA e dos demais direitos humanos, como também assumir suas obrigações, estabelecidas tanto internacional quanto nacionalmente.

A responsabilidade da realização do DHAA é de todos os membros da sociedade. Entretanto, é a sociedade civil organizada nacional, um dos setores que mais tem contribuído para o avanço do processo da garantia do DHAA e o estabelecimento da política de SAN no Brasil, apesar dos grandes entraves políticos e operacionais, oriundos do modelo de desenvolvimento vigente no País. Vários são os desafios e, dentre estes, está o de aproximar e integrar o desenho das políticas públicas de combate à fome à abordagem baseada em direitos.

\section{O significado de uma política pública baseada em direitos}

Ao assumir o pressuposto de que a política funciona como uma ponte entre as questões e/ou demandas públicas e as soluções específicas para tais demandas, é de esperar que tal vinculação possa trazer impactos importantes sobre o desenho 
das políticas públicas ${ }^{16}$, uma vez que as mesmas estão localizadas no domínio da política e, portanto, inseridas na estrutura do Estado e comprometidas com suas obrigações.

Tradicionalmente, as políticas sociais no Brasil, de combate à pobreza e à fome, foram marcadas por um forte caráter assistencialista e clientelista ${ }^{17,18}$. E, apesar de na década de 1990 ter-se iniciado um processo de reorganização das políticas sociais, inspirado na Constituição Federal de 1988, este ainda apresentou, até 2003, uma abordagem que destacava o papel da filantropia e da solidariedade social. Assim, a provisão de serviços e a oferta de bens caracterizavam-se pela redução e dispersão do Estado (portador de obrigações) de suas responsabilidades em relação aos mais pobres (titulares de direitos) e também pelo fortalecimento de parcerias com setores da sociedade civil.

A partir do governo Lula, iniciou-se uma nova fase de reaproximação do Estado de suas responsabilidades de prover direitos, com a concepção de que o DHAA deveria ser garantido pelo Estado a todos os cidadãos. Tal perspectiva motivou a elaboração de uma proposta mais ampla no campo das políticas sociais, relacionadas à segurança alimentar e combate à pobreza. Essa proposta articulava ações estruturantes (voltadas a combater as causas da fome), ações específicas (visando a garantir acesso direto à alimentação) e ações locais (que tinham o objetivo de garantir o abastecimento alimentar no âmbito municipal $)^{17}$. Sem dúvida alguma, essa transição foi o começo de mudança para um novo paradigma. Entretanto, existem ainda alguns componentes que necessitam ser incorporados, para que a aproximação/integração das políticas públicas da abordagem baseada nos direitos aconteça de forma mais efetiva.

Um deles é a incorporação dos princípios da dignidade humana, do imperativo de prestação de contas, da transparência, da não discriminação, da participação e do empoderamento. Tais princípios podem contribuir significativamente para a elaboração de um desenho de política mais ajustado às demandas dos titulares de direito, com maior poder emancipatório, com um controle social mais efetivo e com menos clientelismo.

Em um sistema democrático, espera-se que, além do poder público, outros atores sociais participem ativamente do debate e da definição do desenho das políticas públicas ${ }^{16,17}$. É essa diversidade de atores que faz emergir as diferentes perspectivas e interesses. É por este motivo que, somente a partir do empoderamento da sociedade, o controle social poderá ser exercido em toda a sua plenitude e a correlação de forças, entre os diferentes atores sociais, exercida com mais igualdade.

Tem sido dito que as ações afirmativas têm o objetivo de corrigir distorções históricas ${ }^{11}$, construídas ao longo de uma trajetória de desenvolvimento marcada por um modelo, centrado no mercado e na concentração de renda, que é perverso e excludente. Entretanto, também tem sido argumentado que a manutenção da política macroeconômica não permitirá a eliminação da desigualdade$^{7}$, nem a plena realização dos direitos, uma vez que o foco é o mercado/capital e não o ser humano.

Outro componente além da inclusão dos princípios, é que a abordagem do indivíduo deve ser feita à luz do seu reconhecimento enquanto sujeito de direito, com toda a sua peculiaridade e especificidade, e não mais de forma genérica e abstrata ${ }^{11}$. Quando o indivíduo é compreendido como um sujeito sócio-histórico-cultural, ele não é tratado como um mero objeto de política, e sim como um titular de direitos, para o qual seus direitos fundamentais têm de ser assegurados.

Em que pese a necessidade de políticas sociais que focalizem prioritariamente os grupos mais excluídos, a fim de diminuir a desigualdade, está também em evidência a demanda de maximizar a eficiência de tais ações, bem como aumentar a participação da comunidade.

Assim sendo, é preciso combinar as estratégias da SAN com os processos sociais fundados nos princípios que regem os direitos humanos e o DHAA. 
Por isso, reafirma-se a urgência de consolidar não somente a questão da cultura de avaliação de políticas públicas, especialmente as de combate à fome, mas também de aproximar e integrar os princípios dos direitos humanos no planejamento das políticas de SAN, a fim de reorientar as formas de planejamento, desenho, implantação e análise. O sentido é considerar que, na tríade estrutura-processo-resultado, a estrutura e o processo de uma política ou programa públicos, são fatores que interferem no resultados ${ }^{18}$, e também que, "na perspectiva dos direitos humanos, o processo (como é feito) é tão importante quanto o resultado (o que é feito)" ${ }^{\text {. }}$.

Além disso, torna-se necessário incluir, no contexto tanto da estrutura quanto do resultado a ser alcançado, a promoção do DHAA, para que seja possível também avaliar se a estrutura discursiva do programa, aliada ao processo de transformar em ação e os resultados observados, foram os propostos na estrutura e condutivos à realização do DHAA.

É preciso operacionalizar avaliações de políticas públicas de segurança alimentar e combate à fome, que utilizem matrizes multidimensionais, às quais também estejam integrados os princípios que regem a abordagem baseada nos direitos humanos e que reconheçam o indivíduo como sujeito de direitos.

\section{A insegurança alimentar na perspectiva do DHAA}

Apesar de o conceito estar estabelecido, ainda há muito a ser feito para que a formulação, a execução e o controle social de uma Política Nacional de Segurança Alimentar e Nutricional sejam efetivos. Por exemplo, sabe-se que o conceito de Segurança Alimentar articula, principalmente, duas dimensões: a dimensão alimentar, que diz respeito à produção e à disponibilidade de alimentos, e a dimensão nutricional, que diz respeito às relações entre o ser humano e o alimento ${ }^{8}$. É fundamental que haja um massivo investimento na formulação de políticas públicas que gerem significativas mudanças estruturais e também que a política estratégica para a SAN esteja norteada pelos princípios do DHAA, uma vez que este direito está intrinsecamente ligado à dignidade humana e à justiça social.

\section{Os diferentes enfoques de acessar a SAN e os seus significados}

Conhecer o significado, a magnitude e o impacto que a (in) segurança alimentar pode trazer para vida das pessoas é essencial para o planejamento e a implantação das estratégias da política para SAN.

Historicamente, as avaliações da condição de segurança alimentar de grupos populacionais ou de ações governamentais que lidavam com a fome, tratavam de identificar os padrões de consumo alimentar (e fontes de acesso ao alimento) e os padrões nutricionais manifestos como respostas $^{19}$.

Os dados sobre consumo alimentar eram gerados a partir da folha de balanço alimentar, que é um instrumento publicado anualmente pelo governo de um país, o qual mostra os níveis médios de consumo alimentar (calorias médias por pessoa por dia), identifica as prioridades gerais para análise de consumo e a preocupação global da política alimentar.

Os padrões nutricionais consideravam, principalmente, a avaliação antropométrica de crianças menores de cinco anos (estado nutricional expresso por peso ao nascer, peso para idade, peso para altura e altura para idade). O estado nutricional de um indivíduo é um marcador não específico do seu estado geral de saúde e estado de crescimento. O exame antropométrico avalia o impacto da (in) segurança alimentar e nutricional, porque o foco é a interpretação da expressão plástica do corpo humano. O conceito de plasticidade, que é uma das palavras-chave da biologia humana, trata de dimensionar a magnitude das respostas adaptativas relativas à interação do ser 
humano ao meio ambiente em que vive ${ }^{20}$. A informação antropométrica, portanto, apresenta forte correlação com as múltiplas dimen-sões da saúde e crescimento e seus determinantes sócioeconômicos e ambientais 12,13,19,20.

Entretanto, a evolução do conceito da segurança alimentar com a incorporação da dimensão nutricional e o reconhecimento do DHAA, trouxe repercussões também para os novos enfoques a serem adotados, tanto nas formas de acessar a segurança alimentar e nutricional quanto nos tipos de informações a serem geradas.

A SAN é elemento do cotidiano de todas as pessoas, independentemente do nível socioeconômico do qual façam parte. Entretanto, é na pobreza que a insegurança alimentar, especialmente para os famintos, se expressa como ausência de qualquer expectativa de viver ${ }^{7}$.

A condição de pobreza e a vivência da fome não permitem ao ser humano senão o contato com um padrão de consumo alimentar de natureza insuficiente e inadequada. $\mathrm{O}$ ato de comer é mais do que uma ação com repercussões biológicas, é também uma importante expressão de linguagem, um ato social impregnado de simbolismos. Assim, a escolha do alimento reflete um uso social, que tem um aspecto simbólico. Nesse sentido, o alimento ou os itens alimentares não somente possuem diferentes valorações em termos de hierarquia e prestígio, como também são identificados enquanto alimentos em relação a um sistema de idéias. O alimento e a forma como é utilizado ou disposto na constituição do cardápio revelam uma forma de comunicação e um sistema na estrutura de cada refeição, que traduz uma variedade de informações de extrema importância para sua compreensão de mundo ${ }^{21}$.

No contexto da alimentação, a nutrição é toda a condição que gera vida e também um discurso sobre o real, uma linguagem do real. A insegurança alimentar, especialmente quando se expressa pela fome, rompe toda essa rede de articulações e significados e atinge uma das mais importantes dimensões do ser humano, que é sua própria identidade sociocultural. É nesse sentido que Freitas ${ }^{22}$, a partir de um estudo etnográfico da fome realizado em Salvador, Bahia, afirma que "os significados da fome engendram um texto específico para a compreensão do fenômeno, o qual é percebido como uma doença, mas, antes, revela-se como um estado de desvalorização social do sujeito perante o mundo, a sociedade. Uma desvalorização no sentido ôntico, pois ele não só se sente humilhado, como também se percebe sem qualquer esperança de sair das condições sociais em que vive".

Por outro lado, em determinados contextos, nos quais as condições sociais não são relacionadas à expressão da forma de viver no cotidiano, a restrição alimentar pode se expressar como resposta ao medo de adoecer e morrer.

Um estudo com um grupo de trabalhadores das áreas rurais de Cansanção (semi-árido baiano) encontrou que a exposição à seca constituía-se em um determinante importante da cultura alimentar local. Entretanto, no imaginário da população, a seca não passava pelas determinações sociais, e sim pela relação com a vontade divina e com as condições climáticas ${ }^{23}$.

A inclusão dos aspectos da cultura sobre alimentação e nutrição na análise de políticas sociais, que lidam com este tema é um dos passos para viabilizar o resgate do ser humano no contexto da SAN ${ }^{7}$.

Estudos recentes, sobre a segurança alimentar no Brasil, já utilizaram uma metodologia que incorporava a necessidade de refletir os diferentes níveis e possibilidades de acesso aos alimentos $^{24-27}$. Com isso, adicionou-se um elemento importante, que foi a consideração do componente psicológico da insegurança, pelo reconhecimento do receio ou do medo de sofrer insegurança alimentar na cotidianeidade, ou como possibilidade em um futuro próximo.

É preciso que novos estudos busquem a compreensão sobre o significado dos impactos recebidos pelo ser humano, tanto na dimensão psicológica quanto na física, durante o processo de mediação da (in) segurança alimentar, seja na perspectiva individual, seja no universo familiar. 
No Brasil, a família tem sido o foco para a implantação de determinados programas sociais, porque existe já um reconhecimento de que a família é uma instância potencializadora das ações governamentais, e sujeito capaz de maximizar recurso ${ }^{28,29,30}$. Entretanto, a família pobre, em situação de insegurança alimentar, torna-se vulnerável, podendo representar um espaço de privação, de instabilidade e de esgarçamento dos laços afetivos e de solidariedade ${ }^{25,29}$

A família é o locus para a concretização da proteção integral dos filhos e dos demais membros. É também o próprio substrato de sua identidade social e o espaço para maturação do sentimento de pertencimento, de humanização e desociabilização das pessoas 28,31 .

É nesse sentido que a condição de vulnerabilidade familiar na pobreza, não somente contribui para a sua desestruturação como também a impossibilita potencializar, em alguns contextos, as ações propostas por meio de políticas sociais.

\section{CONSIDERAÇÕ ES FINAIS}

É fundamental revisar o desenho das políticas públicas com abordagens conservadoras, distanciadas dos princípios dos direitos humanos e que não reconhecem a família, nem o indivíduo, como sujeitos de direitos. Também será importante o planejamento de pesquisas capazes de avaliar o impacto que determinados programas sociais possam causar sobre a situação e a percepção da segurança alimentar em famílias que participam dos mesmos.

A busca dos diversos significados da insegurança alimentar contribuirá para o resgate do ser humano como sujeito sócio-histórico-cultural e não apenas como objeto da política pública. Assim, também haverá a mudança de foco do alimento para o ser humano e a priorização da dignidade humana, razão principal pela qual as estratégias de Segurança Alimentar e Nutricional devem ser direcionadas e integradas à abordagem de direitos humanos.

\section{A GRADECIMENTOS}

À Coordenação de Aperfeiçoamento de Pessoal de Nível Superior, pela bolsa de pós-doutorado outorgada (2006-2007) e ao Norwegian Centre for Human Rights, University of Oslo.

\section{REFER Ê N CIAS}

1. Eide WB. From food security to the right to food. In: Eide WB, Kracht $\mathrm{U}$, editors. Food and human rights in development: legal and institutional dimensions and selected topics. Oxford: Intersentia; 2005. v.1.

2. Valente FLS. Do combate à fome à segurança alimentar e nutricional: o direito à alimentação adequada. In: Valente $\mathrm{F}$, editor. Direito humano à alimentação: desafios e conquistas. São Paulo: Cortez; 2002.

3. Eide $W B, K$ racht $U$. The right to adequate food in human rights instruments: legal norms and interpretations. In: Eide WB, Kracht U, editors. Food and human rights in development: legal and institutional dimensions and selected topics. Oxford: Intersentia; 2005. v.1.

4. United Nations. International Covenant on Economic, Social and Cultural Rights (CESCR), United Nations, 16 Dec. 1996. Available from: <http://www.ohchr.org/english/law/cescr.htm>.

5. Comentário Geral número 12: o direito humano à alimentação (art. 11). [acesso 2007 out 24]. Disponível em: <http://www.abrandh.org.br/ downloads/Comentario12.pdf>.

6. Brasil. Lei Orgânica de Segurança Alimentar e Nutricional. Texto Final. 2006 [acesso 2007 out 27] . Disponível em: <http://www.abrandh.org.br/ downloads/losanfinal15092006.pdf>.

7. Freitas MCS, Pena PGL. Segurança alimentar e nutricional: a produção do conhecimento com ênfase nos aspectos de cultura. Rev Nutr [periódico na internet]. 2007 [acesso 2007 nov 6]; 20(1):69-81. Disponível em: <http://www.scielo.br/pdf/rn/v20n1/ a08v20n1.pdf>. doi: 10.1590/S1415-52732007 000100008.

8. Valente FLS, Burity V, Franceschini T, Carvalho MF. Curso formação em direito humano à alimentação adequada. Módulo I. Brasília: ABRANDH; 2007.

9. Valente FLS, Beghin N. Realização do direito humano à alimentação adequada e a experiência brasileira: subsídios para a replicabilidade. Brasília: FAO; 2006. [acesso 2007 out 22]. Disponível em: <http://www.abrandh.org.br/downloads/dha abrasil.pdf $>$.

10. Valente FLS. The human right to food movement in Brazil. In: Eide WB, Kracht U, editors. Food and 
human rights in development: evolving issues and emerging applications. Oxford: Intersentia; 2007.

11. Piovesan F. Ações afirmativas da perspectiva dos direitos humanos. Cad Pesquisa [periódico na internet]. 2005 [acesso 2007 set 10]; 35(124):4355. Disponível em: <http://www.scielo.br/pdf/cp/ v35n124/a0435124.pdf>. doi: 10.1590/S010015742005000100004.

12. Comentário Geral número 3: a natureza das obrigações dos Estados-partes. [acesso 2007 out 15] . Disponível em: <http://www.dhnet.org.br/ dados/cursos/dh/br/pb/dhparaiba/2/c3.html>.

13. Eide A. State Obligations revisited. In: Eide WB, Kracht $U$, editors. Food and human rights in development: evolving issues and emerging applications. Oxford: Intersentia; 2007.

14. Rosa S, Dutschke M. Child rights at the core: the use of international law in South African cases on children's socio-economic rights. SAJHR. 2006; 22(2):224-60.

15. Benevides MV. Educação em direitos humanos: de que se trata? 2000. [acesso 2007 out 15]. Disponível em: <http://www.dhnet.org.br/dados/ boletins/edh/br/boletim1.html\#Dequesetrata>.

16. Lahera, E. Política y políticas públicas. In: Naciones Unidas, editora. Serie: Políticas Sociales. 2004; p.3-32. [acesso 2007 set 22]. Disponível em: <http:// www.eclac.cl/publicaciones/xml/5/19485/ sps95_lcl2176p.pdf>.

17. Instituto de pesquisa econômica aplicada. Assistência social e segurança alimentar. Boletim de Políticas sociais: acompanhamento e análise. [periódico na internet] 13:77-114. (Edicão especial). [acesso 2007 nov 15]. Disponível em:<http:// www.ipea.gov.br/sites/000/2/publicacoes/ bpsociais/bps_13/BPS_13_completo.pdf>.

18. Santos SMC, Santos LMP. Avaliação de políticas públicas de segurança alimentar e combate à fome no período de 1995-2002: abordagem metodológica. Cad Saúde Pública. 2007; 23(5):2029-40. doi: 10.1590/S0102-311X2007000500005.

19. Timmer PC, Falcon WP, Pearson SR, editores. Análise da política alimentar. Londres: Banco Mundial; 1983 [acesso 2007 out 13]. Disponível em: <http:/ /www.ifpri.org/portug/pubs/books/timmer.pdf>.

20. Strickland S, Tuffrey V. Form and function. Cambridge: Smith-Gordon; 1997.

21. Murcott A. Sociological and social anthropological approaches to food and eating. World Rev Nutr Diet. 1988; (55):1-35.

22. Freitas MCS. Uma abordagem fenomenológica da fome. Rev Nutr [periódico na internet]. 2002 [acesso 2007 nov 6]; 15(1):53-69. Disponível em: <http://www.scielo.br/pdf/rn/v15n1/a07v15 n1.pdf>. doi: 10.1590/S1415-52732002000100 007.
23. Assis AMO, Freitas MCS, Oliveira TC, Prado MS Sampaio LR, Machado AD et al. Bró, caxixe e ouricuri, estratégias de sobrevivência no semi-árido baiano. Rev Nutr [periódico na internet].1999 [acesso 2007 nov 6]; 12 (2):159-66. Disponível em: <http://www.scielo.br/pdf/rn/v12n2/v12n2 a05.pdf>. doi: 10.1590/S1415-5273199900020 0005.

24. Fávaro T, Ribas DLB, Zorzatto JR, Segall-Corrêa AM, Panigassi G. Segurança alimentar em famílias indígenas Teréna, Mato Grosso do Sul, Brasil. Cad. Saúde Pública [periódico na internet]. 2007 [acesso 2007 out 30]; 23(4):785-93. Disponível em: <http:// www.scielo.br/pdf/csp/v23n4/05.pdf>. doi: 10.15 90/S0102-311X2007000400006.

25. Sampaio MFA, Kepple AW, Segall-Corrêa AM, Oliveira JTA, Panigassi G, Maranha LK, et al. (In) Segurança Alimentar: experiência de grupos focais com populações rurais do Estado de São Paulo. Segurança Alimentar e Nutricional [periódico na internet]. 2006 [acesso 2007 out 29]; 13(1):64-77. Disponível em: <http://www.unicamp.br/nepa/ arquivo_san/inseguranca_alimentar.pdf $>$.

26. Marin-León L, Segall-Corrêa AM, Panigassi G, Maranha LK, Sampaio AFA, Pérez-Escamilha R. A percepção de insegurança alimentar em famílias com idosos de Campinas, São Paulo, Brasil. Cad Saúde Pública [periódico na internet]. 2005 [acesso 2007 nov 1]; 21(5):1433-40. Disponível em: <http:// www.scielo.br/pdf/csp/v21n5/16.pdf>. doi: 10.15 90/S0102-311X2005000500016.

27. Segall-Corrêa AM, Pérez-Escamilla R, Maranha LK, Sampaio MFA, Yuyuma L, Alencar F, et al. Projeto: acompanhamento e avaliação da segurança alimentar de famílias brasileiras: validação de metodologia e de instrumento de coleta de informação. Relatório Técnico. [acesso 2007 nov 2]. Disponível em: <http://www.opas.org.br/sistema/arquivos/ vru_unic.pdf $>$.

28. Gomes MA, Pereira MLD. Família em situação de vulnerabilidade social: uma questão de políticas públicas. Ciênc e Saúde Coletiva. 2005; 10(2):357-63.

29. ROSE, DD. Interventions to reduce household food insecurity: a synthesis of current concepts and approaches for Latin America. Rev Nutr [periódico na internet]. 2008 [acesso 2009 nov 11]; 21 supl 0. Disponível em <http://www.scielo.br/scielo. php?script=sci_arttext $\&$ pid $=$ S1415-52732008 $000700014 \& \operatorname{lng}=e n \& n r m=i s o>$.

30. Segall-Correa, Marin-Leon L, Helito H, Pérez-Escamilla $R$, Santos LMP, Paes-Sousa R. Transferência de renda e segurança alimentar no Brasil: análise dos dados nacionais. Rev Nutr. 2008; 21 (supl 0):39s-51s.

31. Petrini JC. Pós-modernidade e família. Bauru: Edusc; 2003.

Recebido em: 12/12/2007 Aprovado em: 25/3/2009 